\title{
Optimal power demand curve: a case study in Universiti Putra Malaysia
}

\begin{abstract}
The necessitation in the optimal power demand model, make it an impetus to present this work as a glaring path in determining the optimum power demand curve, using the available data of a substation. The data are used to find the equation and its coefficients of determination that can give the best-fit curve of the power demand of a substation, based on the available data. This will produce a model that can be validated by comparing the data and figured out the equation.
\end{abstract}

Keyword: Modelling; Optimal fit; Optimization; Power demand curve; Substation 